\title{
Preliminary studies on occurrence of middle-season rice high temperature damage in Southwest China in past 50 years
}

\author{
Wang Yan ${ }^{1, a}$, Fan $\mathrm{Li}^{2 *, \mathrm{~b}}$, He Yongkun ${ }^{2}$ \\ ${ }^{1}$ Chongqing Academy of Agricultural Sciences, Jiulongpo District, Chongqing City, 401329, China \\ ${ }^{2}$ Chongqing Institute of Meteorological Sciences, Yubei District, Chongqing, 401147, China \\ a email: x_wangyan@163.com, bemail: fanli_0223@163.com \\ ${ }^{*}$ Corresponding Author
}

Keywords: Middle-season rice; High temperature damage; IDW; Morlet wavelet analysis

\begin{abstract}
Frequent high temperature has become an important agricultural meteorological disaster to the middle-season rice yield and quality in southwest China where has hot rainy season and obviously three-dimensional climate. This paper analyses the meteorological data of more than 70 weather stations in southwest China from 1960 to 2010 using binomial nine moving average method, Morlet wavelet analysis and inverse distance weighted interpolation method, and summarizes the spatial-temporal variation rules of the high temperature damage of middle-season rice from heading and flowering time to grain filling and milky ripe time. The results grasped the macroscopic laws of high temperature damage for rice occurred in southwest, which has the certain reference function for the guidance of high temperature damage prevention for middle-season rice in southwest china, optimizing the layout of rice production.
\end{abstract}

\section{Introduction}

Southwest China is one of the areas with the most complex topography in the world. Lower from north to south and from west to east, the mountain three-dimensional climate significantly enrich the heat, rain and hot season, good conditions for the growth and development of rice, but frequent high temperature of Southwest often threatens rice production, which becomes the region's major agro-meteorological disaster of affecting the rice yield and quality ${ }^{[1-2]}$. As in 2006, the southwest region experienced the unprecedented serious high temperature and drought disasters, and the high temperature damage occurred in the critical period for heading-stage, flowering and grain filling of rice. Sustained high temperature damage and drought resulted in the rice production reduction with a proportion more than $25 \%$ in the area, more than $50 \%$ in local area, and even no harvests. In the process of the whole rice growth and development, the flowering period is the most sensitive to high temperature, followed by filling stage. In the heading and flowering period, high temperatures will affect anther dehiscence, pollen viability, pollen tube growth inhibition and normal shedding, resulting in poor fertilization and increased shell unfilled grains ${ }^{[3-5]}$. In the filling stage, high temperatures will shorten the grain filling period, leading to increased rates of unfilled grains, grain weight decreased, in particular, it will cause loose rice, broken rice rate and chalkiness increase, rice qualitative deterioration ${ }^{[6-7]}$.

In this paper, we use the GIS technology, and try to utilize the Morlet wavelet analysis methods to research the representatives of the meteorological weather station data in the southwest region during the last 50 years, which has important significance on the spatio-temporal distribution of high temperature damage for reproductive middle-season rice, analyzing the extent of occurrence, formulating scientific defensive measures, reducing or avoiding the effect of high temperature and heat harm on rice yield and quality. 


\section{Materials and Methods}

\section{Study Materials}

In this study, data from representative stations in southwest China from 1961 to 2010 during heading and flowering and grain filling period including the average temperature and other meteorological data were analyzed. Based on the principles that the ecological distribution is relatively uniform and meteorological data is relatively complete a total of 70 were selected meteorological stations (Fig 1).

\section{Methods}

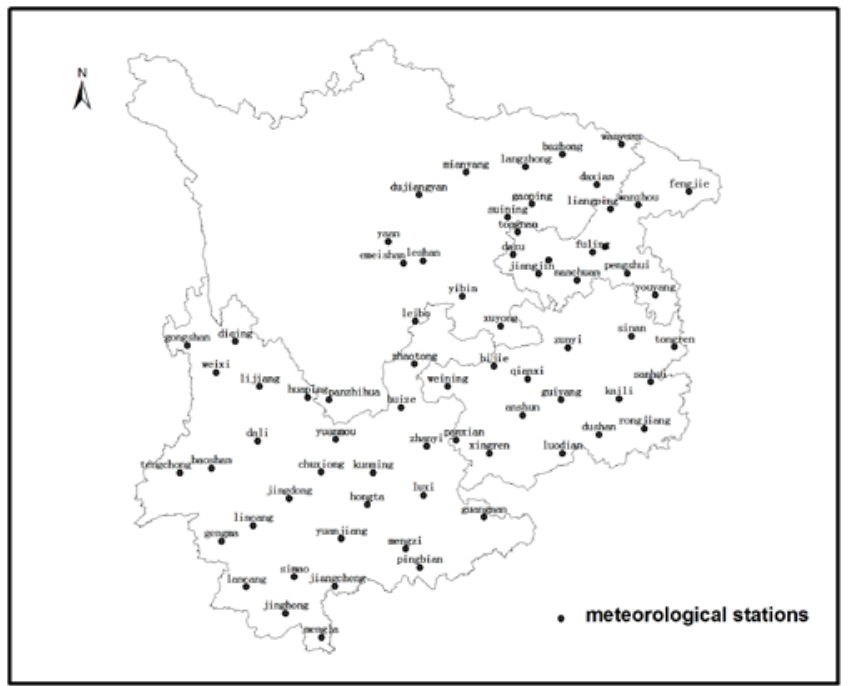

Fig.1. Meteorological stations of the rice high temperature damage in Southwest

\section{1) Indicator determination}

Numerous studies on the high temperature damage to the middle-season rice generally considered that the heading and flowering - filling milk stage is the most sensitive period. And the middle-season rice would be no longer fertilized when the temperature reaches $30 \mathrm{C}$. Furthermore, 35C was been considered as the relatively significant obstacle to high temperatures[8]. It has been widely accepted by most of scholars that a day temperature was defined as daily maximum temperature35C and daily mean temperature30C; One of high temperature damage would be considered to emerge when over 3 days of high temperatures continuously happened. Different degrees of high temperature disaster level can be divided according to the duration of the high temperature [9-14]. And the same indicators were also been applied in this study.

Tab 1. Grading of the high temperature-induced damage index for rice in Southwest China

\begin{tabular}{cccc}
\hline Grade & Slight & Moderate & Severe \\
\hline Tmax & $\geq 35 \mathrm{C}$ & $\geq 35 \mathrm{C}$ & $\geq 35 \mathrm{C}$ \\
Tmean & $\geq 30 \mathrm{C}$ & $\geq 30 \mathrm{C}$ & $\geq 30 \mathrm{C}$ \\
$\mathrm{d}$ & $3-5$ & $6-8$ & $>8$ \\
\hline
\end{tabular}

\section{2) Zoning and determining the cell growth period}

In the current research, altogether four provinces including Yunnan, Guizhou, Sichuan and Chongqing were selected as the study area. The characteristics of topography and climate for these regions are quite different. In the light of the middle-season rice climatic regionalization which was obtained on the basis of Calories, moisture and seasonal conditions, the region of Southwest was classified as the moist two cooked three double cropping of rice cooked with a single. As Sichuan Basin, Guizhou Plateau, northern and southern Yunnan belong to different medium rice climate zones, hence the whole study area was divided into 5 partitions.(1) Eastern Sichuan Basin Zone. In this area, a total of 22 meteorological stations derived from districts of the eastern of Sichuan province, Chongqing and Guizhou province were involved. In addition, the time interval from middle of July to late August was chosen as the analysis period. (2)Western Sichuan Basin Zone. About 7 meteorological stations were involved and the analysis period was from late July to middle of September. (3) North Yunnan and northwest Guizhou dry hot valley Zone. A total of 18 
meteorological stations were involved and the analysis period was from early August to middle of September. (4) Central Yunnan and south-central Guizhou Zone. About 16 meteorological stations were involved and the analysis period was from late August to middle of September. (5) south of Guizhou Zone. About 7 meteorological stations were involved and the analysis period was from late July to middle of August.

\section{3) Analysis method and data processing}

The method of binomial coefficient weighted average method was applied to analysis the inter-annual changes of frequency of high temperature damage. In the spatial pattern study of middle-season rice high temperature damage, the interpolation method of inverse distance weighting (IDW) in ArcGIS10.0 was used for the spatial interpolation of each discrete site. As a result, the value of medium rice of high temperature damage distributing spatially continuous was obtained.

\section{Results}

\section{Time variation}

\section{1)Annual variation}

Different levels of heat damage occurred in 24 basin from the period of semilate rice heading and flowering to Grouting milky maturity in 1960 -2010.The varying pattern of occurrence times was similar to annual days in high temperature damage (Fig.2). High temperature damage appeared the most in 2006 (26 times), flowed by 2010 (21 times) and did not appear in 1987. As shown in the Fig 2 , se- milate rices were damaged seriously by high temper- ature from the period of semilate rice heading and flowering to Grouting milky maturity in 2006, 1994, 1996, 1972, 2010, 2003,1967, 1992, 1976, 1971, and 1978. Among these years accumulated temperature days in 2006 was over 598d, others were over 200d.

Overall trends of slight, moderate and severe high temperature damage varied in different time (Fig.3). Slight high temperature damage occurred annually in 50 years, except in 1987, which rose slightly before 1968, and then felled slowly from 1968 to 1988, fluctuations in the overall fluctuating rose significantly after 90 years (Fig.3a). Moderate high temperature damage did not happen in 1965, 1983, 1993, 1998, 1999, 1999, but overall fluctuations con- tinuously, had a comparatively obvious decline in the $80 \mathrm{~s}$ ((Fig.3b). severe heat damage which did not happen in 21 years changed slowly in 50 years, es- pecially in the first 80 years. The largest annual days of high temperature damage in three levels appeared in 2006.

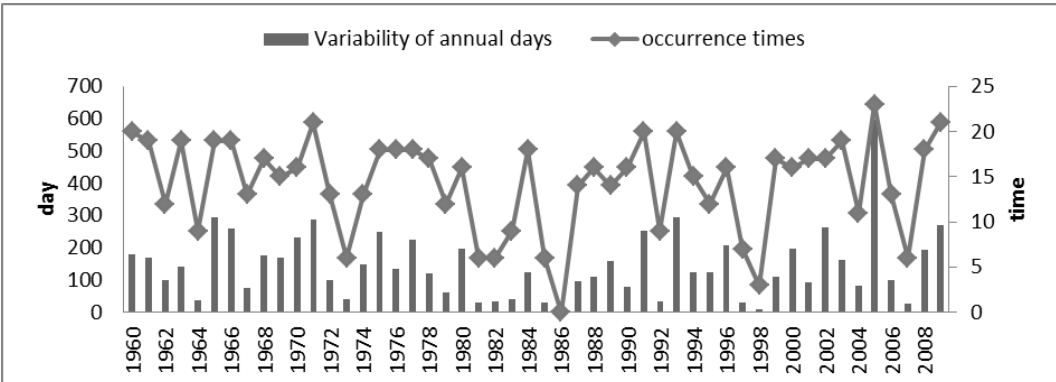

Fig.2. Variability of annual days and occurrence times in high temperature damage during 1960 2010

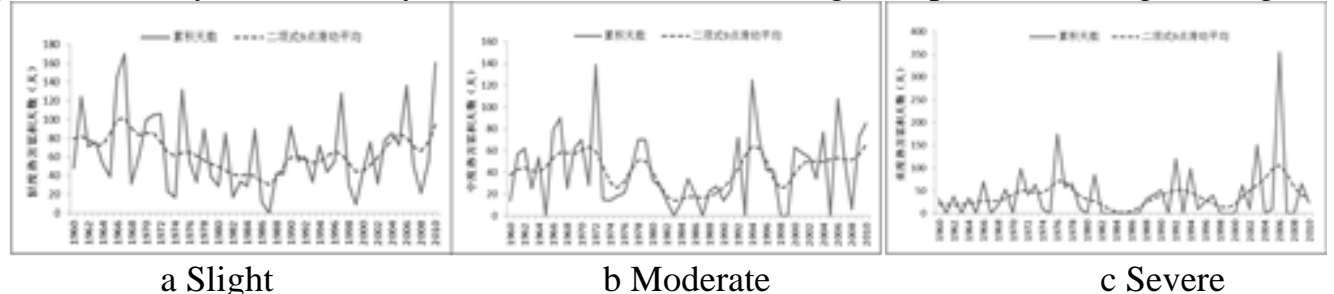

Fig.3. Variability of different grades occurrence days in rice high temperature damage during 1960 2010

\section{2)Interannual variation}

Decadal variability of days and occurrence times in rice high temperature damage during 1960 2010 was shown in Fig 4. As shown in the Fig 4, the overall trend of days and occurrence times varied in an up - down - up - down way. High temperature days and occurrence times maintain a 
higher level in 1960s and 1970s was 153d/a and 15.8 times /a respectively, and declined significantly in 1980s (72.4d/a, 10.3 times /a) which lower than the average of 51 years(144.4d/a, 14.2 times /a), followed by a trend of recovery in $90 \mathrm{~s}(131.3 \mathrm{~d} / \mathrm{a}, 13.2$ times/a), but had yet to reach levels in $60 \mathrm{~s}$ and 70 s. High temperature days increased significantly (190.3d/a) and high temperature damage aggravated (16.2times/a) in the late 20th century.

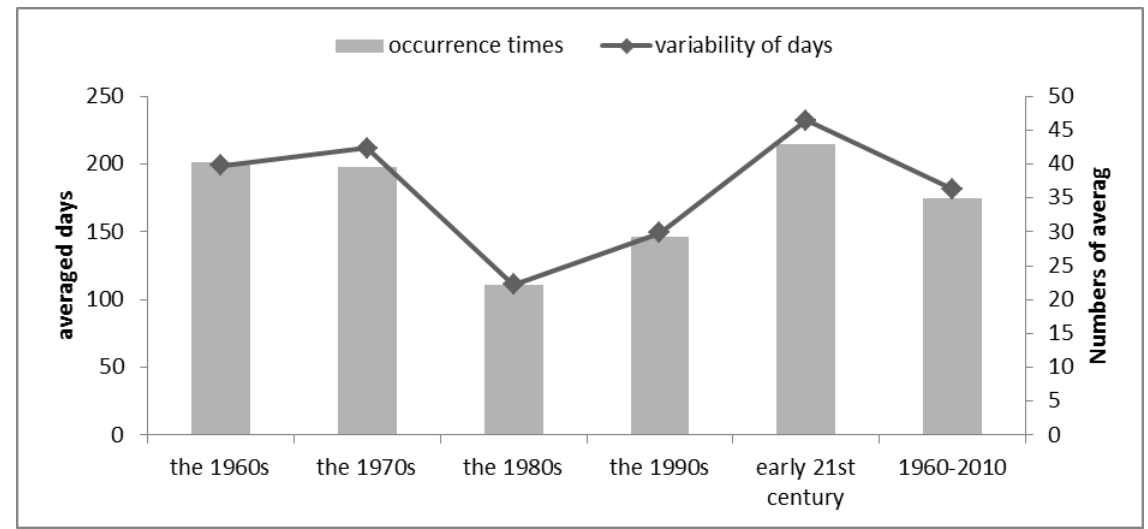

Fig.4. Decadal variability of days and occurrence times in rice high temperature damage during 1960 2010

\section{3)Periodic variation}

Because of good time-frequency localization, it is suitable for wavelet to analysis the local features of period variations in time series. Wavelet analyze is a effective methods for obtaining adjustment law through a complex time series, diagnosing internal hierarchy of climate change, distinguishing the evolution features of time series with different time scales.

The character of the days occurred Middle- season rice high temperature damage with different time scales during recent 50 years in southwest China is shown in Fig 5. A 5-year oscillation is evident in first 15 years with short period of oscillation. The last 35 years exist a 14 -year long period with intense oscillation, which is a major controlling period in middle-season rice high te- mperature damage. There is a 3-year oscillation after 2000. Variables have three most gathered at the center of the energy in the wave energy surface with wavelet domain (Fig 5b), (1) the scale of center is 14 years ,which have 22 years quasi-periodic from 1975 to 2010 and was strongest from 1983 to 2007. (2) The scale of center is 5 years, range from 4 to 6 years. The influence of wave energy is mainly the time domain from 1968 to 1977. (3) The scale of center is around 3years, the influence of time domain is after 2000.

(a)

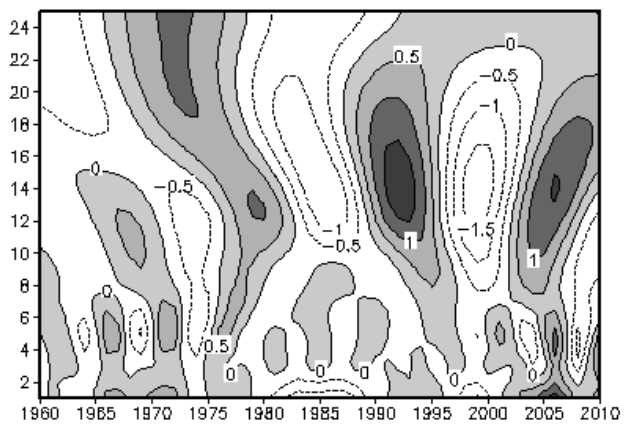

(b)

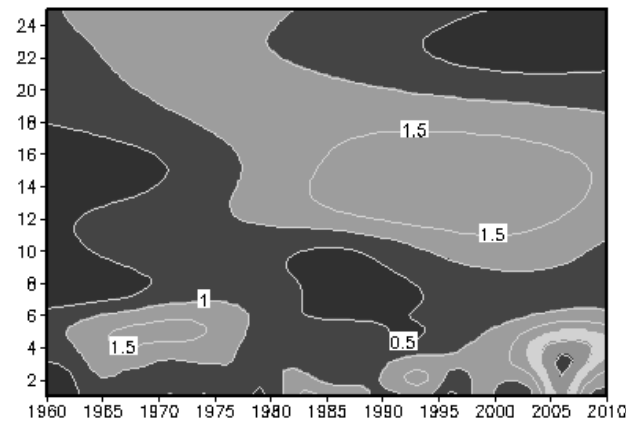

Fig.5. Wavelet transform of rice high temperature damage in 1960 2010(a)and the standardization of wavelet modulus(b)

\section{Spatial variation}

From the spatial distribution of high temperature damage, the cumulative number of days and times on high temperature damage centers on the east Sichuan basin and decrease in all directions. (Fig.6, Fig.7).The 24 sites appear that rice high temperature damage at different levels in 51years with 0.2-9.6 times/10a respectively, and most area occur more than 5times/10a. The cumulative number of days with high temperature damage is $20-100 \mathrm{~d} / 10 \mathrm{a}$ in most area, the high-value region is in the east Sichuan basin, high value centers locate in Jiangjin, Shapingba, Fuling, and Wanzhou. Besides, the nor- theast of Guizhou province occurs frequently, and the less number of days and times 
occurred in central Sichuan basin and the south Yunnan, During head- ing and flowering-filling and seed setting period, there is no high temperature damage in the dry-hot valleys, western, northwestern and eastern Yunnan, southwest of Guizhou.

From the damage degree of high temperature, the range of slight, moderate, severe damage are narrowed from eastern Sichuan basin to Chongqing along Yangtze river with high value center (Fig . 8.).The 24 sites appear slight high temperature damage, most area appear 2.55-7.06 times/10a and high valve center is in the eastern Sichuan basin and northeastern Guizhou, and the highest frequency is 7.06times/10a in Daxian(Fig. 8a).There are 20 cites occur moderate high temperature damage in 1.0- 5.1times/10a, the range of high value have reduced than slight harm and extend from along the Yangtze river to southwest and northeast (Fig. 8b). The fre- quency of severe harm is 0,20-3.92times/10a in 19 sites. High-incidence area scatter across the Jiangjin, Shapingba, Fuling, Wanzhou in Chongqing (Fig.8c).

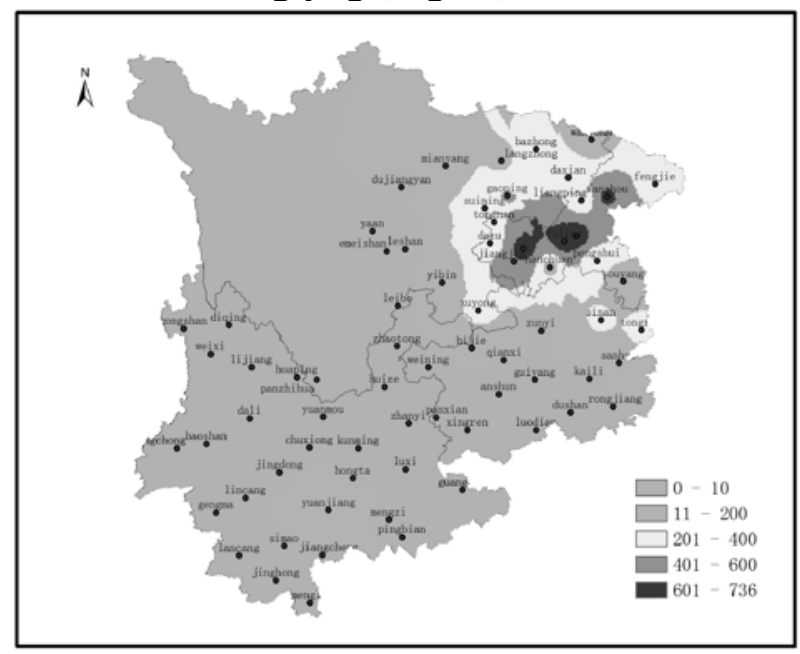

Fig 6

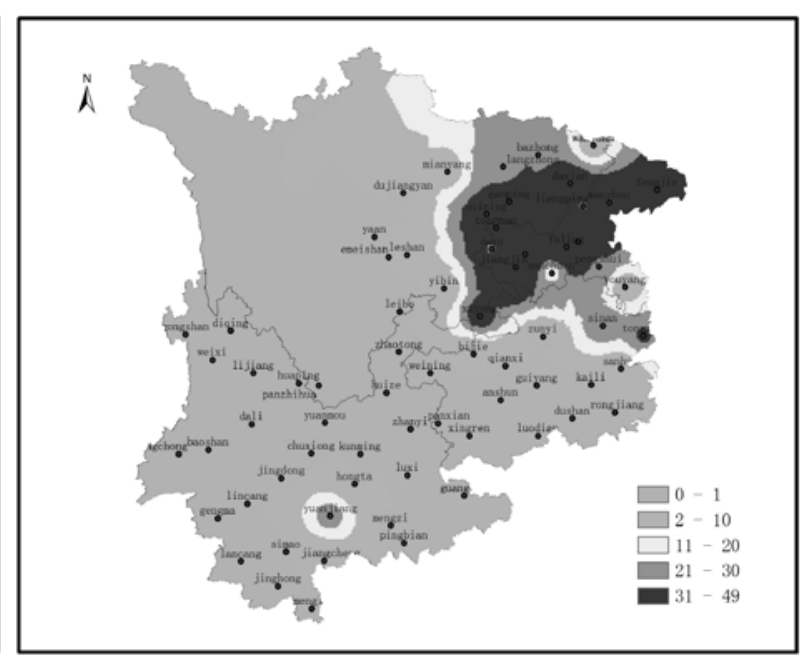

Fig 7

Fig.6. Spatial distribution of occurrence days in rice high temperature damage during 1960 2010

Fig.7. Spatial distribution of occurrence times in rice high temperature damage during 1960 2010

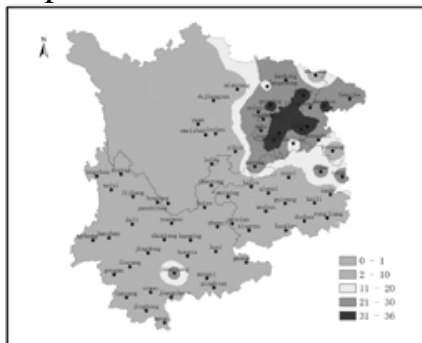

a Slight

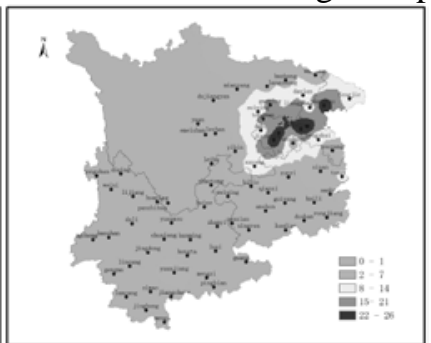

b Moderate

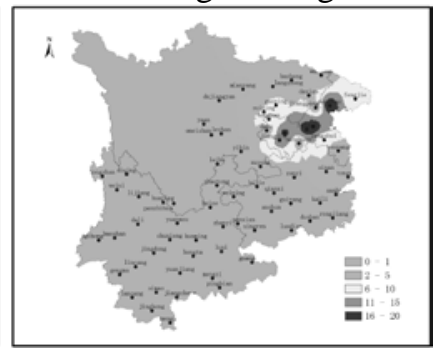

c Severe

Fig.8. Spatial distribution of different grades occurrence times in rice high temperature damage during 1960 2010

\section{Conclusion}

In this paper, considering a continuous 3-day highest daily temperature $\geqslant 35 \mathrm{C}$ and the average temperature $\geqslant 30 \mathrm{C}$ as agricultural meteorological index, which can lead to middle-season rice under high temperature damage, the probability and intensity of high temperature damage in 70 sites of five southwest agricultural areas from the period of the heading and flowering to grain filling were analyzed and we got the following conclusions:

(1) In terms of time changes, from 1960 to 2010, times of rice high temperature damage in the southwest region from the period of the heading and flowering to grain filling and the cumulative number of days, the trend of harm accumulated temperature changes were consistent. Times of rice high temperature damage in 2006 were the maximum, and no high temperature damage happened in 1987. The days of slight high temperature damage occurred fluctuated significantly in the 1990s;

Moderately rice high temperature damage fluctuated continuously, and had a comparatively 
obvious decline in the 1980s; The fluctuation of severe high temperature damage was relatively small in 50 years. Especially, changes before 1980s were relatively flat.

(2) On the spatial distribution, in the southwest, the eastern Sichuan basin had the high value of middle-season rice high temperature damage, and high value center appeared along the Yangtze river in Chongqing. The cumulative number of days of high temperature damage happened were less in the dry-hot valleys (DHV), central Sichuan basin, and central Yunnan, and no high temperature damage happened in other place such as western Yunnan, eastern and northwestern Yunnan, the southwest of Guizhou, etc.

(3) Using wavelet transform, cyclical swings and mutation point features of rice high temperature damage in the southwest nearly 50 years happening in different time scale has been analysised. The previous 15 years had an about 5-year cycle, and in the 35 years later there was a 14-years cycle, which was the mainly controls the cycle. There was an about 3-year oscillation cycle after 2000.

\section{References}

[1].He Y K, Fan L, Yang Y Y. Study on the Occurrence of High Temperature-Induced Heat Damage in Rice in the East of Sichuan Basin in the Past 50 Years [J]. Journal of southwest University (Natural Science Edition), 2011, 33(12): 39-43.

[2].Luo Z Z, Yang Y Y, Tang Y X, et. al., Study on Characteristics of Heat Damage on Rice in Chongqing in Context of Climatic Change[J]. Journal of southwest agricultural, 2011, 24(6):2185-2189.

[3].Mackill D J, Coffinan W R, Rutger J N. Pollen shedding and combining ability for high temperature tolerance in rice[J]. Crop Sci, 1982, 22:730-733.

[4].Matsui T, Kobayasi K, Kagata H, et al. Correlation between viability of pollination and the length of basal dehiscence of the theca in rice and a hot-and-humid condition[J]. Plant Prod Sci, 2005, 8(2):109-114.

[5].Li C D. Analysis on a lot of rice shell caused by high temperature[J]. Shaanxi Journal of Agricultural Sciences, 2003, 5: 45-47.

[6].Huang Y J, Zhang H Y, Guo J Y et al. The preliminary study of physiological mechanism and breeding application of middle-season rice resistance to high temperature. Science Technology and Engineering, 2004, 4(8):655-658.

[7].Resurrection, A P. Effect of temperature during ripening on grain quality of rice[J]. Soil Science and Plant Nutrition, 1977, 23(1):109-112.

[8].Gao S H, Wang P J, et al. High temperature thermal damage and effects on rice in the middle and lower reaches of the Yangtze river[M]. Meteorological press, Beijing 2009:7-8. (in Chinese)

[9].Sen G G N, Research work on Cohen stress resistance genetic breeding of middle-season rice[J]. Hybrid rice. 1992, (1):47-48.

[10].Zhang Y C, He W X, LIi S K. Introduction to Agrometeorological Disaster in China [M]. Beijing: Meteorology, 1991:272-282, 348-353.

[11].Tan H Z, Lan T Y, Ren C F, et al. Studies on high temperature injury on hybrid rice at flowering time and the strategy to avoid high temperature damage[J]. Journal of crops, 1985:103-108.

[12].Wang S K, Wang G W, Wang Y J. Investigation of high temperature thermal pollution in middle-season rice in 2003[J]. Anhui agriculture notification, 2004, 10(1):27, 35.

[13].Yang T M, Chen J H, Effects of Hot Disaster of HighTemperature in Summer on Rice Growth in Central Anhui. Anhui agricultural science, 2007, 35(27):8530-8531.

[14].Hou W F, Wang Q Q, The Change Characteristics of Surface Air Temperature between Changjiang River and Nanling Mountain in Recent 50 Years. Plateau meteorological, 2004, 23(3):400-406. 